\title{
Alzheimer's Disease Research: Scientific Productivity and Impact of the Top 100 Investigators in the Field
}

\author{
Aaron A. Sorensen* \\ Collexis Holdings, Inc., Life Science Solutions Leader, Europe, Via Bramantino 1, 6600 Locarno, Switzerland
}

\begin{abstract}
The online availability of scientific-literature databases and natural-language-processing (NLP) algorithms has enabled large-scale bibliometric studies within the field of scientometrics. Using NLP techniques and Thomson ISI reports, an initial analysis of the role of Alzheimer's disease (AD) within the neurosciences as well as a summary of the various research foci within the AD scientific community are presented. Citation analyses and productivity filters are applied to post-1984, AD-specific subsets of the PubMed and Thomson ISI Web-of-Science literature bases to algorithmically identify a pool of the top AD researchers. From the initial pool of AD investigators, top-100 rankings are compiled to assess productivity and impact. One of the impact and productivity metrics employed is an $\mathrm{AD}$-specific $\mathrm{H}$-index. Within the $\mathrm{AD}$-specific $\mathrm{H}$-index ranking, there are many cases of multiple AD investigators with similar or identical H-indices. In order to facilitate differentiation among investigators with equal or near-equal $\mathrm{H}$ indices, two derivatives of the H-index are proposed: the Second-Tier H-index and the Scientific Following H-index. Winners of two prestigious AD-research awards are highlighted, membership to the Institute of Medicine of the US National Academy of Sciences is acknowledged, and an analysis of highly-productive, high-impact, AD-research collaborations is presented.
\end{abstract}

Keywords: Alzheimer's disease, amyloid- $\beta$, amyloid- $\beta$ protein precursor, citation, citation analysis, H-index, highly-cited, history of science, neurodegenerative diseases, oxidative stress, Scientific following H, scientometrics, Second-tier H-index, tau

\section{INTRODUCTION}

The field of Alzheimer's disease (AD) research, which in 2006 marked its centennial, has progressed at a rapid rate since the late 1970s [1]. Considering the broader field of neuroscience [2], of the estimated 135,000 actively publishing scientists worldwide, roughly $18 \%$ (or 25,000 ), have published one or more papers on $\mathrm{AD}$. This ratio of $\mathrm{AD}$ scientists to all neuroscientists remains constant at 19\% when assessing the 100 most-cited neuroscientists during the period 1997-2007 [3]. In spite of the increasing share of neuroscience activity focusing on $\mathrm{AD}$, there has not been

\footnotetext{
* Corresponding author: Aaron A. Sorensen, Collexis Holdings, Inc., Life Science Solutions Leader, Europe, Via Bramantino 1, 6600 Locarno, Switzerland. E-mail: sorensen@collexis.com.
}

a comprehensive, objective analysis of $\mathrm{AD}$ research through scientometrics [4].

\section{METHODS}

The following three dimensions were selected as a basis to measure the work in AD by individual investigators: total citations, total publications, and $\mathrm{H}-$ index $[5,6]$. The underlying data used in the tabulation of each dimension originate from MEDLINE and Thomson ISI Web of Science.

Two selection filters were used in determining which papers would contribute to an individual scientist's metrics. The first filter was a requirement that all papers to be included in the analysis mention $\mathrm{AD}$ at least once in the Title, Abstract, or Key-Words sections. The second filter is temporal: only papers published between 1 
January 1985 and 21 April 2008 were considered. It is important to recognize the effects of the temporal filter as researchers who made fundamental contributions to the field prior to 1985 but have since slowed in the areas of impact and productivity will be underrepresented in this study due to the 1985 limitation. George Glenner, with his 1984 amyloid-beta-protein discovery, is a case in point. Also of importance is the AD-specific filter, as some prominent AD scientists have strong interests in other areas. Their impact and productivity in nonAD fields will not be recognized in this analysis. Mark Mattson of the National Institute on Aging (with a dual appointment at Johns Hopkins University) is a prime example of this phenomenon.

While the underlying paper-level data were provided by MEDLINE and ISI, the Author-specific tabulations of number of papers, total citations, and $\mathrm{H}$-index were achieved by querying the Collexis-Thomson Alzheimer Dashboard [7]. This tool facilitates author-level bibliometric analysis within a given scientific area [8]. Full names for the AD researchers in this study were derived from the publically-available BiomedExperts repository, which allows the accurate extraction of full names (i.e., last name, first name, middle initial) through author-disambiguation algorithms [9]. While powerful, it was clear during the analysis that the automated, author-disambiguation routines used in assigning papers to the individual scientists are not perfect. The metrics, therefore, represent a good approximation of impact and productivity rather than an exact measurement. The productivity and impact of some scientists, due to variations in the spelling of their last names, are unavoidably underrepresented.

BiomedExperts.com was also utilized in determining each scientist's main line of investigation. The top five $\mathrm{MeSH}$ terms from each scientist's research profile in BiomedExperts were considered. The highest-ranked $\mathrm{MeSH}$ term within the top five for a given scientist that could be considered an AD line-of-investigation was chosen as the main line of investigation for the researcher in question. It is important to note that the papers used to generate the top five MeSH terms for each scientist were not restricted to those papers mentioning $\mathrm{AD}$, but were taken from a collection of PubMed papers representative of an investigator's entire corpus of published work. It follows, then, that the line of investigations chosen for each researcher is not necessarily the line of investigation most frequently found within that scientist's AD papers. The MeSH term chosen is, however, one that the scientist has applied to their $\mathrm{AD}$ research and, more importantly, is the line of investigation most representative of the entire research portfolio of the scientist in question. This approach to line-of-investigation identification has as its goal the assignment of a single MeSH term that describes what a given scientist "brings to bear" within the world of AD research without attempting to compare competing $\mathrm{MeSH}$-term frequencies within an investigator's ADspecific paper corpus.

As part of the $\mathrm{H}$-index analysis, two new metrics are proposed. The Scientific Following $\mathrm{H}(\mathrm{sfH})$ is exactly the same as the traditional $\mathrm{H}$ index except for the fact that the numbers generated are at the author, as opposed to the paper, level. In other words, a scientist has an $\mathrm{sfH}$ of 7 if there are at least 7 investigators who have cited that scientist's work at least seven times (considering all papers of the scientist for whom the SFH is being calculated as well as all papers of the all investigators who have ever cited any paper of the scientist in question). The sheer number of papers needed to calculate the $\mathrm{sfH}$ means that it cannot be tallied by hand, but requires a powerful author-disambiguation algorithm working on a comprehensive citation database. The high-level numbers used to calculate the sfH were generated by a Collexis author-disambiguation algorithm running on top of the raw, ISI Web-of-Science citation data from 1 January 1985 to 1 May 2008. The Second-Tier Hindex (SeTH) is defined as the recalculation of an author's H-index after removing the papers belonging to the author's H-Core [10]. SeTH can be viewed as the author's H-Core pipeline and may be used to predict the rate of increase of an author's H-Index rank within the $\mathrm{AD}$ community in the near term.

As a measure of the acceptance of a given researcher within the AD scientific community, the winners of the MetLife Alzheimer Award for Medical Research [11] and the Potamkin Prize for Research in Pick's, Alzheimer's, and Related Diseases [12] are acknowledged. Membership to the Institute of Medicine of the US National Academy of Sciences is also noted.

Finally, a co-author analysis of the collaboration patterns of the 25 top-ranked scientists (in terms of $\mathrm{H}$ index) was conducted on the MDLogix VisuaLyzer [13] platform using co-author relationships extracted from Thomson WoS and biomedexperts.com

\section{RESULTS}

A brief analysis of the field of $\mathrm{AD}$ research was conducted with the underlying goal of understanding its importance first within the field of neurodegenerative diseases and subsequently within the greater realm of neuroscience research. 
The role of $A D$ within the fields of neurodegenerative diseases and neuroscience

In order to get a rough idea of the place that $\mathrm{AD}$ research holds within neurodegenerative investigation, the Collexis clustering algorithms that are part of the Mediator knowledge discovery platform [14] were utilized to create a semantic grouping of all disease-andpathologic-process-related MeSH terms that have appeared within PubMed papers on neurodegenerative diseases from 1996 to the present. In order to keep the cluster to a reasonable size, an arbitrary threshold of the top 30 terms was set. The ranking of the terms within the neurodegenerative cluster was based on number of PubMed papers containing the term in question. The top five terms in the cluster are, in descending order of number of PubMed papers, Cerebrovascular Accident, Alzheimer Disease, Dementia, Parkinson Disease, and Atrophy.

A similar process was undertaken to identify the top $\mathrm{MeSH}$ concepts within the AD literature. The semantic analysis of the AD-specific literature was conducted using the Thomson-Collexis AD Dashboard. Table 1 contains the top $30 \mathrm{MeSH}$ terms (along with paper counts) for the AD literature base (within PubMed/WoS since 1985). Some of these, such as "mice" and "pathology," are throw-away terms that do not reveal much about the directions of mainstream AD research. Other terms, such as "Amyloid $\beta$-Protein Precursor," "Risk Factors," and "Phosphorylation," give some indication of the prevalence of various lines of investigation within the AD research community.

$\mathrm{AD}$ is the second most mentioned MeSH term within the neurodegenerative literature. Of note is the fact that the MeSH categories are not mutually exclusive. Many papers on neurodegeneration will contain a number of the above concepts. For instance it is not uncommon for a paper on $\mathrm{AD}$ to mention atrophy and dementia.

Moving beyond the neurodegenerative diseases to neuroscience as a whole, the Thomson ISI Neuroscience Journal Citation Report was used to define which journals (and subsequently which papers and, by extension, which authors) should be included in a bibliometric overview of the field. The list of neuroscience journals is updated by Thomson ISI every year. The most recent version (2007) lists 211 journals as falling squarely within the realm of neuroscience.

Thomson ISI Web of Science (WoS) was queried for the number papers published by all 211 journals for the year 2007 . The query returned 40,801 papers. A technical clarification is that a Thomson query restricts the
Table 1

Shows, in descending order, the top 30 terms (and paper counts) for MESH terms in the AD literature

\begin{tabular}{lr}
\hline Dementia & 17,836 \\
Amyloid & 12,188 \\
Rats & 7,715 \\
Mice & 7,694 \\
Genes & 7,307 \\
Risk & 5,675 \\
Amyloid $\beta$-Protein Precursor & 5,550 \\
Aging & 5,483 \\
Pathology & 5,340 \\
Parkinson Disease & 4,464 \\
Genetics & 4,231 \\
Apolipoproteins E & 4,020 \\
Mutation & 3,831 \\
Risk Factors & 3,416 \\
Antibodies & 3,190 \\
Alleles & 3,128 \\
Oxidative Stress & 2,921 \\
Death & 2,900 \\
Cell Death & 2,675 \\
Apoptosis & 2,388 \\
Screening & 2,369 \\
Phosphorylation & 2,338 \\
Mice, Transgenic & 2,274 \\
Dementia, Vascular & 2,239 \\
tau Proteins & 2,211 \\
Cerebrovascular Accident & 2,148 \\
Atrophy & 2,145 \\
Amyloid $\beta$-Protein & 2,116 \\
Protein Isoforms & 2,093 \\
Syndrome & 2,033 \\
\hline & \\
&
\end{tabular}

end user to a maximum of 50 Boolean terms per query resulting in a total of five queries (i.e., 50 journals per query) needed to calculate the total output of the neuroscience journals for 2007. Using Thomson's advanced analysis feature, for each resulting query, an estimate of the number of unique authors who collaborated on the papers in question was generated. The Thomson estimate for the total number of authors collaborating on the 40,801 papers appearing in the 211 journals in 2007 was 135,649. This number will be inflated by the double-counting of authors as the 50-booleanterm restriction limits the effectiveness of the Thomson author-disambiguation algorithms in tabulating author counts. The inflation in the author count is likely offset by the fact that the list of 211 journals cannot be considered comprehensive. The list does not, for instance, include journals such as The Lancet or The New England Journal of Medicine, which, while not considered to be neuroscience journals per se, surely publish many articles each year by neuroscientists. Another factor that offsets the inflation inherent in the Thomson-based estimate is the fact that in any given year, there may be active neuroscientists who did not publish a single pa- 
Table 2

Most Prolific AD Investigators from 1 January 1985 through 21 April 2008 using a combined (minus duplicate papers) PubMed and WoS AD paper count (institutional affiliation and ALZForum ID indicated)

\begin{tabular}{|c|c|c|c|c|}
\hline Rank & Author & Institutional affiliation & $\begin{array}{l}\text { ALZForum profile ID (to view } \\
\text { profile, append ID to following base } \\
\text { URL: http://www.alzforum.org/com/ } \\
\text { res/detail.asp?id= }\end{array}$ & $\begin{array}{c}\text { PubMed + } \\
\text { Thomson } \\
\text { WoS } \\
\text { papers on } \\
\text { AD (1985- } \\
2008) \\
\end{array}$ \\
\hline 1 & Winblad, Bengt & Karolinska Institute & $\begin{array}{l}\text { \{E43DF156-ED34-4D58-BF6C- } \\
\text { 0877090A0298\} }\end{array}$ & 575 \\
\hline 2 & Perry, George & University of Texas at San Antonio & $\begin{array}{l}\text { \{AF7F6C7C-A7F3-4E6D-96BB- } \\
\text { 6BF5FA53F1AE }\end{array}$ & 516 \\
\hline 3 & Smith, Mark A & Case Western Reserve University & $\begin{array}{l}\{\text { EB2B9380-C464-4652-A816- } \\
1511461825 B 9\}\end{array}$ & 405 \\
\hline 4 & Morris, John C & Washington University & $\begin{array}{l}\{99215 F D 7-E E 99-46 A A-B A 1 E- \\
\text { E268A3519A30\} }\end{array}$ & 384 \\
\hline 5 & Masters, Colin L & University of Melbourne & & 381 \\
\hline 6 & Cummings, Jeffrey L & University of California, Los Angeles & $\begin{array}{l}\{8 \mathrm{C} 11591 \mathrm{E}-4 \mathrm{E} 55-4 \mathrm{AE} 5-94 \mathrm{FC}- \\
1 \mathrm{~B} 971473592 \mathrm{~F}\}\end{array}$ & 366 \\
\hline 7 & Hyman, Bradley T & Harvard University & $\begin{array}{l}\{84 \mathrm{BB} 57 \mathrm{D} 7-3 \mathrm{CF} 3-4171-\mathrm{ABD} 5- \\
\text { 52EDDC50D0F5 }\end{array}$ & 365 \\
\hline 8 & Beyreuther, Konrad & University of Heidelberg & & 355 \\
\hline 9 & Hardy, John & $\begin{array}{l}\text { Institute of Neurology, University } \\
\text { College London }\end{array}$ & $\begin{array}{l}\{609139 A 5-33 D A-47 C 0-80 A 8- \\
39008 C C 32589\}\end{array}$ & 351 \\
\hline 10 & Lee, Virginia M-Y & University of Pennsylvania & $\begin{array}{l}\text { \{40D45C85-D2AB-4CE4-9B0A- } \\
\text { 43987940E6F8\} }\end{array}$ & 337 \\
\hline 10 & Trojanowski, John Q & University of Pennsylvania & $\begin{array}{l}\text { \{A64BEA2F-3B60-44E8-8234- } \\
\text { 1D04B74A86B8 }\}\end{array}$ & 337 \\
\hline 12 & Masliah, Eliezer & University of California, San Diego & & 324 \\
\hline 13 & Blennow, Kaj & University of Göteborg & $\begin{array}{l}\text { \{0CF2EA98-1EAB-49B3-9EC7- } \\
\text { 3F3C06A761DB }\}\end{array}$ & 322 \\
\hline 14 & Soininen, Hilkka & University of Kuopio & $\begin{array}{l}\{\mathrm{B} 4939 \mathrm{E} 15-\mathrm{F} 8 \mathrm{FD}-4 \mathrm{~F} 2 \mathrm{~B}-\mathrm{AB} 64- \\
\text { DD997AD4D1A0 }\}\end{array}$ & 312 \\
\hline 15 & Selkoe, Dennis J & Harvard University & $\begin{array}{l}\text { \{6380DD6F-FE33-486A-849D- } \\
\text { 7A0C29F9D11F }\}\end{array}$ & 310 \\
\hline 15 & Stern, Yaakov & Columbia University & & 310 \\
\hline 17 & Thal, Leon J & University of California, San Diego & $\begin{array}{l}\text { \{4E295BE2-A736-4C92-B3F1- } \\
\text { C2EDD637C81D }\}\end{array}$ & 307 \\
\hline 18 & Dickson, Dennis & Mayo Clinic - Florida & & 296 \\
\hline 18 & Mayeux, Richard & Columbia University & $\begin{array}{l}\{95988961-9 E 7 A-4 F A A-9175- \\
67 F F 33 \text { CAD981 }\}\end{array}$ & 296 \\
\hline 20 & Scheltens, Philip & Vrije Universiteit Amsterdam & & 295 \\
\hline 21 & DeKosky, Steven T & University of Virginia & $\begin{array}{l}\{\text { E5C66A9A-A258-415C-8BC5- } \\
\text { 0296A5C } 8519 C\}\end{array}$ & 291 \\
\hline 22 & Mattson, Mark P & $\begin{array}{l}\text { National Institutes on Aging/John Hopkins } \\
\text { University }\end{array}$ & $\begin{array}{l}\{26 \mathrm{C} 09 \mathrm{C} 83-\mathrm{AE} 3 \mathrm{~A}-44 \mathrm{CB}-\mathrm{B} 141- \\
\text { 499C7B06C7F9 }\end{array}$ & 287 \\
\hline 23 & Iqbal, Khalid & New York State Institute of Basic Research & $\begin{array}{l}\text { \{7A550DCA-F8C5-4F69-BB7D- } \\
\text { F4D8A2FDE1C8 }\}\end{array}$ & 273 \\
\hline 23 & Markesbery, William R & University of Kentucky & & 273 \\
\hline 25 & Petersen, Ronald C & Mayo Clinic - Minnesota & $\begin{array}{l}\{5 \mathrm{~B} 456 \mathrm{~F} 01-5380-4 \mathrm{FA} 7-80 \mathrm{D} 4- \\
\text { 1CFF6DEBE61B }\}\end{array}$ & 269 \\
\hline 26 & Miller, Bruce L & University of California, San Francisco & & 259 \\
\hline 27 & Cotman, Carl W & University of California, Irvine & $\begin{array}{l}\{961 \mathrm{C} 9734-8 \mathrm{~A} 09-42 \mathrm{~EB}-\mathrm{B} 6 \mathrm{E} 8- \\
\text { D53B8E57F0D4 }\}\end{array}$ & 256 \\
\hline 28 & Bennett, David A & Rush University Medical Center & $\begin{array}{l}\{\mathrm{C} 7893 \mathrm{C} 4 \mathrm{E}-7 \mathrm{~F} 1 \mathrm{E}-466 \mathrm{D}-\mathrm{AFD} 2- \\
\text { 468F845193CE }\}\end{array}$ & 253 \\
\hline 29 & Rossor, Martin N & Institute of Neurology, London & & 252 \\
\hline 30 & Roses, Allen D & Duke University & & 248 \\
\hline 30 & Tanzi, Rudolph E & Harvard University & $\begin{array}{l}\{\mathrm{BE74EFC0-7CCD-4285-ADA0-} \\
98744 \mathrm{E} 847 \mathrm{CB} 1\}\end{array}$ & 248 \\
\hline
\end{tabular}


Table 2, continued

\begin{tabular}{|c|c|c|c|c|}
\hline Rank & Author & Institutional affiliation & $\begin{array}{l}\text { ALZForum profile ID (to view } \\
\text { profile, append ID to following base } \\
\text { URL: http://www.alzforum.org/com/ } \\
\text { res/detail.asp?id= }\end{array}$ & $\begin{array}{c}\text { PubMed + } \\
\text { Thomson } \\
\text { WoS } \\
\text { papers on } \\
\text { AD (1985- } \\
\text { 2008) }\end{array}$ \\
\hline 32 & Perry, Robert H & University of Newcastle & & 244 \\
\hline 32 & St George-Hyslop, Peter & University of Toronto & & 244 \\
\hline 34 & Cairns, Nigel J & Washington University & $\begin{array}{l}\{197 D 5 D E D-35 D 9-43 C 2-8 F 10- \\
\text { F1833C6729B0 }\}\end{array}$ & 239 \\
\hline 35 & Jellinger, Kurt A & University of Vienna & $\begin{array}{l}\text { \{ECAC7439-DC8C-436D-AB6D- } \\
\text { 28142DEAB970\} }\end{array}$ & 237 \\
\hline 36 & Gauthier, Serge & McGill University & & 234 \\
\hline 37 & Mann, David M A & University of Manchester & $\begin{array}{l}\{\text { 4E8997D2-7CD4-423F-8BE5- } \\
\text { 561501ACC706 }\end{array}$ & 233 \\
\hline 38 & Arai, Heii & Juntendo University & & 231 \\
\hline 39 & Butterfield, D Allan & University of Kentucky & $\begin{array}{l}\{26 \mathrm{~A} 78291-1528-4 \mathrm{C} 14-\mathrm{B} 010- \\
\text { BF1C13AFD864\} }\end{array}$ & 230 \\
\hline 40 & McGeer, Patrick L & University of British Columbia & $\begin{array}{l}\{72 \text { AE9E7E-AAD5-4D44-9F32- } \\
\text { C853AB0376F6 }\end{array}$ & 227 \\
\hline 41 & Wisniewski, Henryk M & NY State Institute of Basic Research & $\begin{array}{l}\{1991 \mathrm{~F} 36 \mathrm{~B}-97 \mathrm{~A} 4-48 \mathrm{~B} 2-\mathrm{B} 894- \\
3518017523 \mathrm{~F} 0\}\end{array}$ & 226 \\
\hline 42 & Hodges, John R & University of Cambridge & & 221 \\
\hline 43 & McKeith, Ian G & Newcastle University & & 218 \\
\hline 43 & Rapoport, Stanley I & National Institute on Aging & & 218 \\
\hline 45 & Frangione, Blas & New York University & $\begin{array}{l}\text { \{8ED69192-D1E1-4E51-92CA- } \\
\text { E95BF09A5B0B }\}\end{array}$ & 215 \\
\hline 46 & Riekkinen, PJ & University of Kuopio & & 213 \\
\hline 46 & Weiner, Michael W & University of California, San Francisco & $\begin{array}{l}\text { \{74BDBD98-E4D8-42AF-9F21- } \\
\text { 5B3E36BC7672\} }\end{array}$ & 213 \\
\hline 48 & Hampel, Harald & University of Dublin & $\begin{array}{l}\{\text { A3072FDB-0B98-4AAA-8B52- } \\
\text { BD4CC7D24487\} }\end{array}$ & 210 \\
\hline 48 & Zhu, Xiongwei & Case Western Reserve University & $\begin{array}{l}\{509699 B 4-2 A 3 F-494 D-8920- \\
\text { D4CBB55D8781 }\}\end{array}$ & 210 \\
\hline 50 & Kurz, Alexander & University of Munich & & 209 \\
\hline 51 & Lannfelt, Lars & Uppsala University & $\begin{array}{l}\{5 \mathrm{C} 19 \mathrm{D} 4 \mathrm{CD}-\mathrm{FA} 3 \mathrm{~F}-4 \mathrm{CCA}-\mathrm{AF} 88- \\
2 \mathrm{~F} 3700 \mathrm{E} 848 \mathrm{C} 0\}\end{array}$ & 208 \\
\hline 52 & Delacourte, André & Inserm & $\begin{array}{l}\{\text { DC4117FC-DD3E-49DF-9A82- } \\
\text { AC19E1C0A75D }\}\end{array}$ & 207 \\
\hline 52 & Nordberg, Agneta & Karolinska Institute & $\begin{array}{l}\{180 B 457 E-96 C F-4 F 7 E-A 764- \\
\text { E46EFE91A5BA }\}\end{array}$ & 207 \\
\hline 54 & Albert, Marilyn S & John Hopkins University & $\begin{array}{l}\text { \{670FF754-CEB5-4B65-910F- } \\
\text { 10F3987AE0F2 }\end{array}$ & 203 \\
\hline 55 & Farlow, Martin R & Indiana University & & 202 \\
\hline 56 & $\begin{array}{l}\text { Pericak-Vance, Margaret } \\
\text { A }\end{array}$ & University of Miami & & 201 \\
\hline 57 & Hofman, Albert & Erasmus MC, Rotterdam & & 200 \\
\hline 57 & Wilson, Robert S & Rush Medical Center & & 200 \\
\hline 59 & Iwatsubo, Takeshi & University of Tokyo & $\begin{array}{l}\text { \{FB389991-D859-4E72-8CBA- } \\
\text { 111EEDEE7152\} }\end{array}$ & 199 \\
\hline 60 & Swaab, Dick F & University of Amsterdam & & 194 \\
\hline 61 & Salmon, David P & University of California, San Diego & & 193 \\
\hline 62 & Price, Donald L & John Hopkins University & $\begin{array}{l}\text { \{EA5E79C9-2531-481F-A2B9- } \\
\text { C390BFBEE188 }\end{array}$ & 190 \\
\hline 63 & Ballard, Clive & King's College London & & 187 \\
\hline 63 & Haass, Christian & Ludwig-Maximilians-University Mïnchen & $\begin{array}{l}\{32 \mathrm{~B} 49 \mathrm{~F} 40-2 \mathrm{~A} 4 \mathrm{~B}-4 \mathrm{D} 08-8097- \\
\text { E48A52277D68 }\end{array}$ & 187 \\
\hline 63 & Perry, Elaine K & Newcastle University & & 187 \\
\hline 63 & Small, Gary W & University of California, Los Angeles & & 187 \\
\hline 67 & Larson, Eric B & University of Washington & & 186 \\
\hline
\end{tabular}


Table 2, continued

\begin{tabular}{|c|c|c|c|c|}
\hline Rank & Author & Institutional affiliation & $\begin{array}{l}\text { ALZForum profile ID (to view } \\
\text { profile, append ID to following base } \\
\text { URL: http://www.alzforum.org/com/ } \\
\text { res/detail.asp?id= }\end{array}$ & $\begin{array}{c}\text { PubMed + } \\
\text { Thomson } \\
\text { WoS } \\
\text { papers on } \\
\text { AD (1985- } \\
2008)\end{array}$ \\
\hline 67 & Saunders, Ann M & GlaxoSmithKline & & 186 \\
\hline 69 & Whitehouse, Peter J & Case Western Reserve University & & 185 \\
\hline 69 & Wilcock, Gordon K & University of Bristol & $\begin{array}{l}\text { \{FDEA1208-6FAB-4F04-B5C0- } \\
\text { EC23770AB48E }\}\end{array}$ & 185 \\
\hline 71 & Growdon, John H & Harvard University & & 183 \\
\hline 71 & O'Brien, John T & Newcastle University & & 183 \\
\hline 71 & Sorbi, Sandro & University of Florence & $\begin{array}{l}\{\text { F5712128-4E65-4730-B87A- } \\
455670822025\}\end{array}$ & 183 \\
\hline 74 & Ikeda, Kenji & Tokyo Institute of Psychiatry & & 182 \\
\hline 74 & Riederer, Peter & Universität Würzburg & & 182 \\
\hline 76 & Davies, Peter & Albert Einstein College of Medicine & $\begin{array}{l}\{\text { DCB4F0CB-E49B-4CA8-AA8D- } \\
\text { 52B7ABECAF6A }\}\end{array}$ & 180 \\
\hline 76 & Vellas, Bruno & Inserm, Toulouse & & 180 \\
\hline 78 & Knopman, David S & Mayo Clinic - Minnesota & & 178 \\
\hline 78 & Sunderland, Trey & National Institute on Aging & & 178 \\
\hline 80 & Davis, Kenneth L & Mt. Sinai & & 172 \\
\hline 80 & Hansen, Lawrence A & University of California, San Diego & & 172 \\
\hline 82 & Goate, Alison M & Washington University & $\begin{array}{l}\{12 \mathrm{EDC} 4 \mathrm{D} 8-4 \mathrm{~B} 4 \mathrm{~B}-4070-\mathrm{BCFD}- \\
682 \mathrm{C} 40 \mathrm{E} 46 \mathrm{C} 8 \mathrm{E}\}\end{array}$ & 171 \\
\hline 82 & Younkin, Steven G & Mayo Clinic - Florida & & 171 \\
\hline 84 & Galasko, Douglas & University of California, San Diego & $\begin{array}{l}\text { \{7BE13DDE-7F10-4209-977C- } \\
\text { F3DDB26D24E4 }\}\end{array}$ & 170 \\
\hline 84 & Goedert, Michel & University of Cambridge & & 170 \\
\hline 86 & Lovestone, Simon & King's College London & & 169 \\
\hline 87 & Avila, Jesús & Universidad Autónoma de Madrid & $\begin{array}{l}\{22 \mathrm{E} 78 \mathrm{~B} 64-\mathrm{C} 398-4396-8 \mathrm{FFE}- \\
\text { 7FD1D13C59B2 }\}\end{array}$ & 167 \\
\hline 87 & Haines, Jonathan L & Vanderbilt University & & 167 \\
\hline 89 & Braak, Heiko & Johann Wolfgang Goethe-Universität & & 166 \\
\hline 89 & Schellenberg, Gerard D & University of Washington & $\begin{array}{l}\text { \{ADFAA4F6-4CFE-4B0E-B226- } \\
\text { 254B305438FD }\end{array}$ & 166 \\
\hline 91 & Fox, Nick C & Imperial College London & $\begin{array}{l}\text { \{BB6AD238-6411-4932-B7E9- } \\
\text { B8B32B8867F2 }\end{array}$ & 164 \\
\hline 91 & Hutton, Michael & Mayo Clinic - Florida & & 164 \\
\hline 93 & Mohs, Richard C & Eli Lilly & $\begin{array}{l}\text { \{6CF5D84E-78BA-46E8-BA98- } \\
\text { B2F4FEC7A81B }\}\end{array}$ & 163 \\
\hline 94 & Jagust, William J & University of California, Berkeley & & 162 \\
\hline 95 & Hof, Patrick R & Mt. Sinai & & 161 \\
\hline 95 & Mufson, Elliott J & Rush University & $\begin{array}{l}\{7 F 9 B 1 A F 5-9253-4214-94 A 7- \\
\text { ABA2BF687B67\} }\end{array}$ & 161 \\
\hline 95 & Schneider, Lon S & University of Southern California & $\begin{array}{l}\{\text { E6787F54-6A48-4124-8151- } \\
\text { 93EE69EF470F }\}\end{array}$ & 161 \\
\hline 98 & Grossman, Murray & University of Pennsylvania & & 160 \\
\hline 99 & Nitsch, Roger M & University of Zurich & $\begin{array}{l}\{8 \mathrm{E} 530 \mathrm{CE} 7-874 \mathrm{~F}-4166-97 \mathrm{E} 6- \\
464130 \mathrm{~F} 3831 \mathrm{D}\}\end{array}$ & 158 \\
\hline 99 & Wallin, Anders & University Göteborg & & 158 \\
\hline
\end{tabular}

per. An example of this phenomenon would be neuroscientists working within a pharmaceutical or biotech company that has placed a publication embargo on their current research. Considering the possible inflation due the double counting of authors but the counteracting nature of the underestimation of total papers and the inability to account for non-publishing neuroscientists in any given year, an estimate of 135,000 active neu- roscientists worldwide is likely a fair estimate of the true number. Knowing the approximate total number of neuroscience papers and authors provides an opportunity to calculate the contribution that $\mathrm{AD}$ makes to the field. Considering that the number of WoS papers published in 2007 that mentioned AD is 7,002 (note: the fact that, when put together, the year and quantity of $\mathrm{AD}$ papers combine to form a numeric palindrome 
Table 3

Most-Cited authors from 1 January 1985 through 21 April 2008 based on citation rates for AD papers appearing in Thomson ISI WoS (the main line of investigation from BiomedExperts for each scientist is also indicated)

\begin{tabular}{|c|c|c|c|}
\hline Rank & Author & $\begin{array}{l}\text { Main line of } \\
\text { investigation }\end{array}$ & $\begin{array}{c}\text { Times cited in Alzheimer's } \\
\text { work since } 1985\end{array}$ \\
\hline 1 & Selkoe, Dennis J & Amyloid $\beta$-Protein & 40,100 \\
\hline 2 & Beyreuther, Konrad & Amyloid $\beta$-Protein Precursor & 25,231 \\
\hline 3 & Roses, Allen D & Apolipoproteins E & 24,500 \\
\hline 4 & Mattson, Mark P & Calcium & 23,704 \\
\hline 5 & Hardy, John & tau Proteins & 23,046 \\
\hline 6 & Masters, Colin L & Amyloid $\beta$-Protein Precursor & 22,768 \\
\hline 7 & Pericak-Vance, Margaret A & Pedigree (genealogy) & 21,638 \\
\hline 8 & Lee, Virginia M-Y & tau Proteins & 21,341 \\
\hline 9 & Tanzi, Rudolph E & Amyloid $\beta$-Protein & 21,144 \\
\hline 10 & Trojanowski, John Q & tau Proteins & 20,190 \\
\hline 11 & Morris, John C & Neuropsychological Tests & 19,938 \\
\hline 12 & Hyman, Bradley $\mathrm{T}$ & Amyloid $\beta$-Protein & 18,864 \\
\hline 13 & Masliah, Eliezer & Nerve Tissue Proteins & 18,636 \\
\hline 14 & Saunders, Ann M & Apolipoproteins E & 17,098 \\
\hline 15 & Cummings, Jeffrey L & Neuropsychological Tests & 16,498 \\
\hline 16 & Perry, George & Oxidative Stress & 16,293 \\
\hline 17 & Cotman, Carl W & Amyloid $\beta$-Protein & 16,198 \\
\hline 18 & Dickson, Dennis & tau Proteins & 15,995 \\
\hline 19 & Goedert, Michel & tau Proteins & 15,974 \\
\hline 20 & Lieberburg, I & Amyloid $\beta$-Protein & 15,863 \\
\hline 21 & Winblad, Bengt & Neuropsychological Tests & 15,157 \\
\hline 22 & Younkin, Steven G & Amyloid $\beta$-Protein & 15,028 \\
\hline 23 & McGeer, Patrick L & Choline O-Acetyltransferase & 14,108 \\
\hline 24 & Smith, Mark A & Oxidative Stress & 13,772 \\
\hline 25 & Price, Donald L & Amyloid $\beta$-Protein Precursor & 13,735 \\
\hline 26 & Petersen, Ronald C & Neuropsychological Tests & 13,588 \\
\hline 87 & St George-Hyslop, Peter & Presenilin-1 & 13,271 \\
\hline 28 & Strittmatter, Warren J & Apolipoproteins E & 13,180 \\
\hline 29 & Thal, Leon J & Choline O-Acetyltransferase & 13,159 \\
\hline 30 & Mayeux, Richard & Apolipoproteins E & 13,081 \\
\hline 31 & Schmechel, Donald E & Apolipoproteins E & 12,900 \\
\hline 32 & Rogers, Jack T & Amyloid $\beta$-Protein Precursor & 12,805 \\
\hline 33 & Multhaup, Gerd & Amyloid $\beta$-Protein Precursor & 12,761 \\
\hline 34 & Haass, Christian & Amyloid Precursor Protein Secretases & 12,758 \\
\hline 35 & Markesbery, William R & Neurofibrillary Tangles & 12,687 \\
\hline 36 & Schenk, Dale & Amyloid $\beta$-Protein & 12,429 \\
\hline 37 & Frangione, Blas & Amyloid & 12,299 \\
\hline 38 & Hofman, Albert & Risk Factors & 12,279 \\
\hline 39 & Haines, Jonathan L & Chromosome Mapping & 12,202 \\
\hline 40 & Sisodia, Sangram S & Amyloid $\beta$-Protein Precursor & 12,037 \\
\hline 41 & Hansen, Lawrence A & Lewy Bodies & 11,900 \\
\hline 42 & Perry, Robert H & Lewy Bodies & 11,753 \\
\hline 43 & Fraser, Paul E & Presenilin-1 & 11,731 \\
\hline 44 & Myers, Richard H & Risk Factors & 11,257 \\
\hline 45 & Miller, Bruce L & Neuropsychological Tests & 11,172 \\
\hline 46 & Stern, Yaakov & Neuropsychological Tests & 11,161 \\
\hline 47 & Rossor, Martin N & Magnetic Resonance Imaging & 10,978 \\
\hline 48 & Goate, Alison M & Presenilin-1 & 10,974 \\
\hline 49 & Growdon, John H & Choline & 10,962 \\
\hline 50 & Beal, M Flint & Disease Models, Animal & 10,616 \\
\hline 51 & Seubert, Peter & Amyloid $\beta$-Protein & 10,524 \\
\hline 52 & Wilson, Robert S & Neuropsychological Tests & 10,452 \\
\hline 53 & Mohs, Richard C & Neuropsychological Tests & 10,131 \\
\hline 54 & Mann, David M A & Neurofibrils & 10,126 \\
\hline 55 & Albert, Marilyn S & Neuropsychological Tests & 10,081 \\
\hline 56 & Lansbury, Peter T & Amyloid & 10,040 \\
\hline 57 & Minoshima, Satoshi & Tomography, Emission-Computed & 9,983 \\
\hline
\end{tabular}


Table 3, continued

\begin{tabular}{|c|c|c|c|}
\hline Rank & Author & $\begin{array}{l}\text { Main line of } \\
\text { investigation }\end{array}$ & $\begin{array}{l}\text { Times cited in Alzheimer's } \\
\text { work since } 1985\end{array}$ \\
\hline 58 & Salmon, David P & Neuropsychological Tests & 9,966 \\
\hline 59 & Koo, Edward H & Amyloid $\beta$-Protein Precursor & 9,744 \\
\hline 60 & Hutton, Michael & tau Proteins & 9,686 \\
\hline 61 & Iqbal Khalid & tau Proteins & 9,530 \\
\hline 62 & Schellenberg, Gerard D & Apolipoproteins E & 9,496 \\
\hline 63 & Davies, Peter & Tau Proteins & 9,493 \\
\hline 64 & Hodges, John R & Neuropsychological Tests & 9,380 \\
\hline 65 & Bird, Thomas D & Pedigree (genealogy) & 9,377 \\
\hline 66 & Butterfield, D Allan & Oxidative Stress & 9,319 \\
\hline 67 & Braak, Heiko & Neurofibrillary Tangles & 9,277 \\
\hline 68 & McKeith, Ian G & Lewy Bodies & 9,208 \\
\hline 69 & Eckman, Christopher B & Amyloid $\beta$-Protein & 9,116 \\
\hline 70 & Perry, Elaine K & Receptors, Nicotinic & 9,032 \\
\hline 71 & Iwatsubo, Takeshi & Amyloid $\beta$-Protein & 8,901 \\
\hline 72 & Farlow, Martin R & Cholinesterase Inhibitors & 8,841 \\
\hline 73 & DeKosky, Steven T & Neuropsychological Tests & 8,776 \\
\hline 74 & Ihara, Yasuo & tau Proteins & 8,768 \\
\hline 75 & Kokmen, Emre & Risk Factors & 8,761 \\
\hline 76 & Bennett, David A & Neuropsychological Tests & 8,734 \\
\hline 77 & Citron, Martin & Amyloid Precursor Protein Secretases & 8,702 \\
\hline 78 & Jellinger, Kurt A & Autopsy & 8,674 \\
\hline 79 & Small, Gary W & Apolipoproteins E & 8,663 \\
\hline 80 & Wisniewski, Henryk M & Neurofibrils & 8,605 \\
\hline 81 & Breteler, Monique M B & Risk Factors & 8,559 \\
\hline 82 & Lannfelt, Lars & Amyloid $\beta$-Protein Precursor & 8,518 \\
\hline 83 & McGeer, Edith G & Choline O-Acetyltransferase & 8,497 \\
\hline 84 & Teplow, David B & Amyloid $\beta$-Protein & 8,284 \\
\hline 85 & Galasko, Douglas & Neuropsychological Tests & 8,259 \\
\hline 86 & Bush, Ashley I & Amyloid $\beta$-Protein & 8,244 \\
\hline 87 & Gusella, James F & Chromosome Mapping & 8,133 \\
\hline 88 & Mandelkow, Eckhard & tau Proteins & 8,106 \\
\hline 88 & Mandelkow, Eva-Maria & tau Proteins & 8,106 \\
\hline 90 & Mirra, Suzanne S & Apolipoproteins E & 8,015 \\
\hline 91 & Soininen, Hilkka & Apolipoproteins E & 8,004 \\
\hline 92 & Gilbert, John R & Polymorphism, Single Nucleotide & 7,916 \\
\hline 93 & Braak, Eva & Neurofibrillary Tangles & 7,905 \\
\hline 94 & Kramer, Joel H & Neuropsychological Tests & 7,882 \\
\hline 95 & Mullan, Michael & Amyloid $\beta$-Protein & 7,835 \\
\hline 96 & Gandy, Samuel E & Amyloid $\beta$-Protein Precursor & 7,767 \\
\hline 97 & Golde, Todd E & Amyloid $\beta$-Protein & 7,729 \\
\hline 98 & Katzman, Robert & Apolipoproteins E & 7,567 \\
\hline 99 & Davis, Kenneth L & Physostigmine & 7,545 \\
\hline 100 & Gauthier, Serge & Cholinesterase Inhibitors & 7,526 \\
\hline
\end{tabular}

does not represent a typographical error) and that the Thomson estimate for actively-publishing AD authors is 24,768 , we can conclude that overall, AD papers represent approximately $17 \%$ of the neuroscience literature and $18 \%$ of neuroscientists.

One way to check the validity of this estimate is to test whether or not the percentages remain true when checked against Thomson's list of the 100 most highly cited neuroscientists for the period 1997-2007 [3]. Each name on the most-highly-cited neuroscientist list was checked against each of the three AD top-100 lists compiled for this study (i.e., most prolific, most-cited, and highest $\mathrm{H}$-index). If an author on the neuroscience list also appeared on any of the three AD lists, they were given an $\mathrm{AD}$ designation. This exercise resulted in the categorization of 19 of the 100 most-cited neuroscientists as being AD researchers. This number confirms the previous approximation that AD investigators make up approximately $18 \%$ of the neuroscience research community.

Productivity and impact among $A D$ investigators - the three metrics

Having established the role AD plays within the area of neurodegenerative and nervous-system diseases, the 
focus of the analysis was turned to understanding the field of $\mathrm{AD}$ research from a scientometric viewpoint with the ultimate objective being that of determining which AD investigators have contributed the most to the field since 1985. A pool of the top 150 AD researchers was generated by consulting biomedexperts.com (a PubMed-based data source) and the Collexis-Thomson WoS Dashboard on AD. Included in the master list of 150 were the most prolific AD authors (based on PubMed and WoS AD paper counts) as well as the most highly-cited authors using WoS AD citation counts. Hindices, total paper counts, and total citations were calculated for all authors. For each category, a top-100 list was generated.

Tables 2, 3, and 5 are the top-100 lists for each category.

\section{Lines of investigation at the summit of the $A D$ scientific community}

Below are the numbers of investigators (of the 150 evaluated) who focus on the various lines of investigation (as listed in Table 3). Although it is clear that certain interrelated lines of investigation might be best combined to form single line of investigation (e.g., Amyloid- $\beta$ Protein, Amyloid $\beta$-Protein Precursor, and Amyloid Protein Precursor Secretases), it was determined that the defining of such composite, line-ofinvestigation categories was beyond the scope of the current study and could lead to bias.

\section{Further refining impact measures - scientific- following $H$}

While the H-index tracks impact by analyzing the body of published work of given investigator, it does not give a clear indication of the thought-leadership position that a given scientist holds within the core community of investigators comprising the research community for a focused field. In other words, the H-index provides an understanding for the overall impact of an investigator but not how concentrated or dispersed that impact is among that investigator's closest scientific peers. In order to assess the scientific following of a given investigator, the scientific-following $\mathrm{H}$-index $(\mathrm{sfH})$ is proposed. The $\mathrm{sfH}$ is calculated in the same way as person's standard H-index, the only difference being that instead of counting the number of papers that have been cited a given number of times, one counts the number of scientists who, in all of their work, have cited a particular investigator in all of that investiga-
Table 4

AD lines of investigation among pool of top 150 researchers

\begin{tabular}{ll}
\hline Neuropsychological tests & 22 \\
\hline Tau Proteins & 20 \\
Amyloid $\beta$-Protein & 16 \\
Apolipoproteins E & 12 \\
Amyloid $\beta$-Protein Precursor & 9 \\
Magnetic Resonance Imaging & 8 \\
Risk Factors & 6 \\
Neurofibrillary Tangles & 5 \\
Choline O-Acetyltransferase & 4 \\
Oxidative Stress & 4 \\
Cholinesterase Inhibitors & 3 \\
Lewy Bodies & 3 \\
Pedigree (genealogy) & 3 \\
Presenilin-1 & 3 \\
Amyloid & 2 \\
Amyloid Precursor Protein Secretases & 2 \\
Chromosome Mapping & 2 \\
Nerve Tissue Proteins & 2 \\
Neurofibrils & 2 \\
Receptors, Nicotinic & 1 \\
Age of Onset & 1 \\
Autopsy & 1 \\
Calcium & 1 \\
Choline & 1 \\
Disease Models, Animal & 1 \\
Electroencephalography & 1 \\
Estradiol & 1 \\
Immunohistochemistry & 1 \\
Longitudinal Studies & 1 \\
Monoamine Oxidase & 1 \\
Nootropic Agents & 1 \\
Nutrition Assessment & 1 \\
Phosphoproteins & 1 \\
Physostigmine & 1 \\
Polymorphism, Single Nucleotide & 1 \\
Positron-Emission Tomography & 1 \\
Tacrine & 1 \\
\hline Vasopressins & 1 \\
\hline & 1 \\
\hline
\end{tabular}

tor's work. In other words, one must simply substitute people, as represented by all of their work, for papers in the $\mathrm{H}$-index calculation. Considering the case of a scientist with an sfH of 5, one would know that there are five investigators (other than scientist under analysis) who have cited (considering all of their papers) that scientist's work (considering all of the scientist's papers) at least five times. Just as the $\mathrm{H}$-index provides lower bounds on the quantity and quality of the entire body of a given scientist's highest impact work, so the sfH provides lower bounds on the quantity and quality of the scientific following, within an investigator's immediate research community, that a given investigator has achieved. For two or more scientists with similar impact measures (i.e., H-index, total citations, or average citation per paper), the $\mathrm{sfH}$ will facilitate an un- 


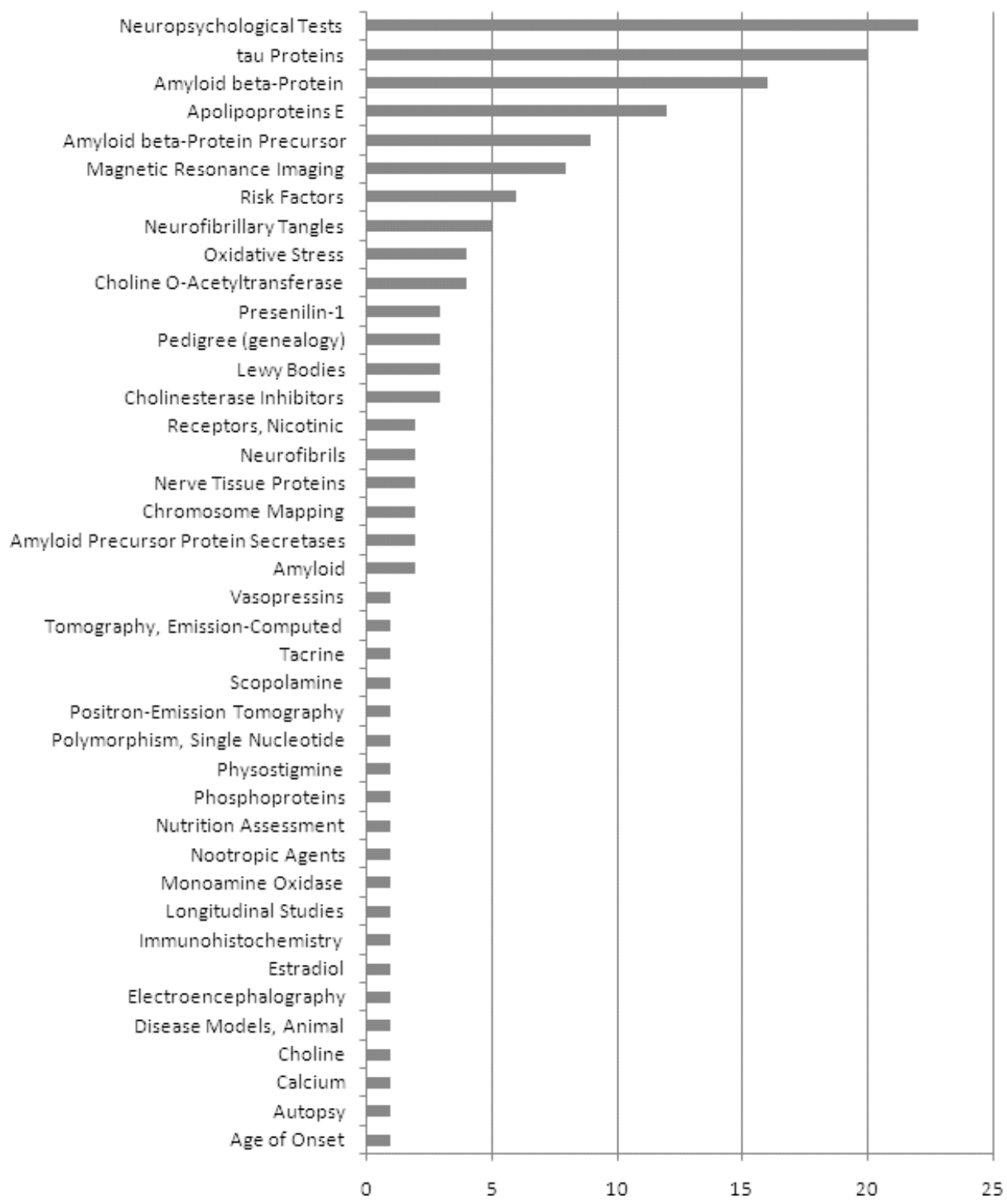

Fig. 1. Distribution of line-of-investigation (based on top MeSH terms in biomedexperts.com) among the top-150 AD researchers.

derstanding of which of them has had the biggest role in school-of-thought leadership. Holding total impact constant, a scientist with a low sfH will have that level of impact spread out across a relatively large number of investigators who may or may not have the same research focus. Again holding total impact constant, a scientist with a high $\mathrm{sfH}$, however, has their impact concentrated in a relatively small number of investigators who typically work in the same area of investigation. In this way, school-of-thought leadership can be inferred through analysis of sfH levels among in- vestigators contributing to the same research area (see Table 5 for sfH scores for the top-100 H-indices).

Thinking about the future - the second-tier H-index

Hirsch has presented compelling arguments that the $\mathrm{H}$-index is an excellent predictor of future impact and productivity [6]. The H-index is of little help, however, in predicting future movement of individuals up and down a ranking in which many of the scientists have identical or nearly-identical Alzheimer H-indices. An 
Table 5

Authors with Highest $\mathrm{H}$-indices calculated from same document base as used for the total-citation tabulations in Tables 2 and 3

\begin{tabular}{|c|c|c|c|c|c|}
\hline Rank & $\begin{array}{l}\text { Author } \\
*=\text { Winner of MetLife Award } \\
\hat{=}=\text { Winner of Potomkin Prize } \\
+=\text { Institute of Medicine member }\end{array}$ & H-index & $\begin{array}{c}\text { Second-tier } \\
\text { H-index }\end{array}$ & $\begin{array}{c}\text { H rank - } \\
\text { SeTH rank }\end{array}$ & $\begin{array}{l}\text { Scientific } \\
\text { following } \\
\text { H-index }\end{array}$ \\
\hline 1 & Selkoe, Dennis J $*^{\wedge}+$ & 102 & 53 & 0 & 29 \\
\hline 2 & Mattson, Mark P & 86 & 48 & 0 & 23 \\
\hline 3 & Lee, Virginia M-Y $*^{\wedge}+$ & 82 & 47 & 0 & 24 \\
\hline 4 & Trojanowski, John $\mathrm{Q}^{* \wedge}+$ & 74 & 45 & 0 & 27 \\
\hline 5 & Beyreuther, Konrad *^ & 72 & 44 & 0 & 24 \\
\hline 5 & Hyman, Bradley $\mathrm{T}^{\wedge}$ & 72 & 42 & -2 & 25 \\
\hline 7 & Masliah, Eliezer & 71 & 40 & -2 & 20 \\
\hline 8 & Morris, John $\mathrm{C}^{\wedge}$ & 70 & 40 & -1 & 27 \\
\hline 9 & Goedert, Michel *^ & 67 & 29 & -23 & 17 \\
\hline 10 & Perry, George & 66 & 42 & 3 & 24 \\
\hline 10 & Tanzi, Rudolph E*^ & 66 & 35 & -6 & 23 \\
\hline 12 & Masters, Colin L^ & 65 & 43 & 6 & 24 \\
\hline 12 & Hardy, John *^ & 65 & 37 & -2 & 37 \\
\hline 12 & Dickson, Dennis * & 65 & 32 & -8 & 24 \\
\hline 12 & Mayeux, Richard ^ + & 65 & 31 & -15 & 23 \\
\hline 16 & Winblad, Bengt & 64 & 40 & 7 & 21 \\
\hline 16 & Cummings, Jeffrey L & 64 & 34 & -2 & 13 \\
\hline 18 & Cotman, Carl W* & 63 & 38 & 6 & 13 \\
\hline 19 & McGeer, Patrick L & 62 & 35 & 3 & 12 \\
\hline 20 & Price, Donald L $* \wedge+$ & 61 & 30 & -10 & 22 \\
\hline 21 & Frangione, Blas *^ & 60 & 37 & 7 & 15 \\
\hline 22 & Smith, Mark A & 59 & 34 & 4 & 26 \\
\hline 23 & Haass, Christian^ & 58 & 32 & 3 & 22 \\
\hline 23 & Stern, Yaakov & 58 & 32 & 3 & 22 \\
\hline 23 & Markesbery, William R & 58 & 31 & -4 & 22 \\
\hline 26 & Roses, Allen D $*^{\wedge}+$ & 57 & 32 & 6 & 17 \\
\hline 27 & Petersen, Ronald $\mathrm{C}^{\wedge}$ & 56 & 29 & -5 & 28 \\
\hline 27 & Thal, Leon $\mathrm{J}^{\wedge}$ & 56 & 28 & -8 & 19 \\
\hline 27 & Sisodia, Sangram $\mathrm{S} * \wedge$ & 56 & 27 & -15 & 21 \\
\hline 27 & Hansen, Lawrence A & 56 & 26 & -21 & 9 \\
\hline 31 & Hofman, Albert & 55 & 28 & -4 & 30 \\
\hline 32 & Mann, David M A & 54 & 28 & -3 & 13 \\
\hline 33 & Perry, Robert H & 53 & 32 & 13 & 14 \\
\hline 33 & St George-Hyslop, Peter $*^{\wedge}+$ & 53 & 25 & -23 & 21 \\
\hline 33 & Younkin, Steven G^ & 53 & 24 & -31 & 15 \\
\hline 36 & Hodges, John R & 52 & 32 & 16 & 20 \\
\hline 37 & Davies, Peter & 51 & 26 & -11 & 21 \\
\hline 38 & Butterfield, D Allan & 50 & 32 & 18 & 20 \\
\hline 38 & Albert, Marilyn S & 50 & 28 & 3 & 17 \\
\hline 38 & Growdon, John H & 50 & 28 & 3 & 11 \\
\hline 38 & Fraser, Paul E & 50 & 25 & -18 & 20 \\
\hline 38 & Beal, M Flint + & 50 & 24 & -26 & 21 \\
\hline 43 & DeKosky, Steven T & 49 & 28 & 8 & 23 \\
\hline 43 & Miller, Bruce L & 49 & 27 & 1 & 23 \\
\hline 43 & Iwatsubo, Takeshi & 49 & 25 & -13 & 22 \\
\hline 43 & Bush, Ashley I^ & 49 & 22 & -30 & 18 \\
\hline 47 & McGeer, Edith G & 48 & 38 & 35 & 11 \\
\hline 47 & Soininen, Hilkka & 48 & 31 & 20 & 17 \\
\hline 47 & Rapoport, Stanley I & 48 & 30 & 17 & 14 \\
\hline 47 & Iqbal, Khalid ^ & 48 & 27 & 5 & 14 \\
\hline 47 & Hof, Patrick R & 48 & 26 & -1 & 13 \\
\hline 47 & Saunders, Ann M & 48 & 25 & -9 & 17 \\
\hline 47 & Larson, Eric B & 48 & 22 & -26 & 13 \\
\hline 47 & Multhaup, Gerd & 48 & 21 & -32 & 18 \\
\hline 55 & McKeith, Ian G & 47 & 29 & 23 & 13 \\
\hline 55 & Rossor, Martin N & 47 & 26 & 7 & 19 \\
\hline
\end{tabular}


Table 5, continued

\begin{tabular}{|c|c|c|c|c|c|}
\hline Rank & $\begin{array}{l}\text { Author } \\
*=\text { Winner of MetLife Award } \\
\hat{=}=\text { Winner of Potomkin Prize } \\
+=\text { Institute of Medicine member }\end{array}$ & H-index & $\begin{array}{c}\text { Second-tier } \\
\text { H-index }\end{array}$ & $\begin{array}{c}\text { H rank - } \\
\text { SeTH rank }\end{array}$ & $\begin{array}{c}\text { Scientific } \\
\text { following } \\
\text { H-index }\end{array}$ \\
\hline 55 & Mohs, Richard C & 47 & 24 & -9 & 9 \\
\hline 55 & Ihara, Yasuo *^ & 47 & 24 & -9 & 2 \\
\hline 55 & Schellenberg, Gerard D*^ & 47 & 23 & -14 & 18 \\
\hline 55 & Koo, Edward $\mathrm{H}$ & 47 & 20 & -30 & 14 \\
\hline 55 & Mandelkow, Eckhard & 47 & 17 & -39 & 12 \\
\hline 55 & Mandelkow, Eva-Maria & 47 & 17 & -39 & 12 \\
\hline 63 & Perry, Elaine K & 46 & 27 & 21 & 10 \\
\hline 63 & Breteler, Monique M B & 46 & 25 & 7 & 24 \\
\hline 63 & Davis, Kenneth L & 46 & 25 & 7 & 12 \\
\hline 63 & Greengard, Paul *+ & 46 & 19 & -26 & 20 \\
\hline 63 & Pericak-Vance, Margaret A ${ }^{+}$ & 46 & 18 & -30 & 24 \\
\hline 68 & Blennow, Kaj & 45 & 27 & 26 & 20 \\
\hline 68 & Delacourte, André & 45 & 26 & 20 & 14 \\
\hline 68 & Wisniewski, Henryk M & 45 & 26 & 20 & 9 \\
\hline 68 & Salmon, David P & 45 & 22 & -5 & 9 \\
\hline 72 & Gandy, Samuel E & 44 & 23 & 3 & 10 \\
\hline 72 & Galasko, Douglas & 44 & 21 & -7 & 9 \\
\hline 72 & Goate, Alison $\mathrm{M}$ *^ & 44 & 20 & -13 & 29 \\
\hline 72 & Kokmen, Emre & 44 & 19 & -17 & 21 \\
\hline 76 & Hutton, Michael ^ & 43 & 24 & 12 & 27 \\
\hline 76 & Braak, Heiko & 43 & 23 & 7 & 16 \\
\hline 76 & Lansbury, Peter T & 43 & 11 & -27 & 20 \\
\hline 76 & Lieberburg, I & 43 & 6 & -28 & 21 \\
\hline 80 & Riekkinen, PJ & 42 & 27 & 38 & 12 \\
\hline 80 & Bennett, David A & 42 & 26 & 32 & 17 \\
\hline 80 & Mufson, Elliott J & 42 & 26 & 32 & 13 \\
\hline 80 & Jellinger, Kurt A & 42 & 25 & 24 & 13 \\
\hline 80 & Sano, Mary & 42 & 20 & -5 & 17 \\
\hline 80 & Bird, Thomas D* & 42 & 19 & -9 & 19 \\
\hline 85 & Swaab, Dick F & 41 & 28 & 51 & 12 \\
\hline 85 & Nordberg, Agneta & 41 & 23 & 17 & 13 \\
\hline 85 & Farlow, Martin R & 41 & 21 & 7 & 10 \\
\hline 85 & Breitner, John C S & 41 & 19 & -3 & 12 \\
\hline 85 & Grundke-Iqbal, Inge & 41 & 16 & -12 & 8 \\
\hline 85 & Teplow, David B & 41 & 15 & -15 & 20 \\
\hline 92 & Jagust, William J & 40 & 22 & 19 & 12 \\
\hline 93 & Cairns, Nigel J & 39 & 25 & 37 & 12 \\
\hline 93 & Smith, Glenn E & 39 & 22 & 20 & 20 \\
\hline 93 & Nitsch, Roger M^ ${ }^{\wedge}$ & 39 & 21 & 14 & 12 \\
\hline 93 & Wilcock, Gordon K & 39 & 20 & 8 & 8 \\
\hline 93 & Braak, Eva & 39 & 17 & -1 & 13 \\
\hline 93 & Rogers, Jack T & 39 & 17 & -1 & 6 \\
\hline 93 & Haines, Jonathan L & 39 & 16 & -5 & 24 \\
\hline 93 & Schenk, Dale^ & 39 & 16 & -5 & 22 \\
\hline 93 & Spillantini, Maria Grazia ^ & 39 & 12 & -9 & 17 \\
\hline
\end{tabular}

example of this is the fact that Dickson, Masters, and Hardy all have an AD H-index of 65 . Using only the $\mathrm{H}$-index, we are unable to discriminate between them in terms of how they might fare in the same ranking if conducted at a future date. In order to address this issue, a new metric called the Second-Tier H, or SeTH is proposed. The SeTH is calculated first by removing from consideration all papers that make up an individual's H-core. The H-core represents all papers that have enough citations to contribute to a given individual's
H-index calculation [10]. Once the H-core has been removed, a scientist's H-index is recalculated. The new $\mathrm{H}$-index is the researcher's Second-Tier H. SeTH can be helpful to discriminate between the future potential of authors with equivalent (or nearly equivalent) $\mathrm{H}$ indices for the simple reason that an individual's SeTH is a quantification of the highly-cited work of a given scientist that is not currently being considered when calculating that person's H-index.

A sports analogy exists in professional baseball in 
the U.S. or professional soccer in many other countries. The major league teams often have one or more affiliated minor league teams with players who do not yet have the skills to play on the top team. Over time, the best players from the minor-league or "farm" teams will make their way to the roster of the major league team. So it is with the papers in SeTH. These papers represent a given author's "minor-league" papers, which, as they gather more citations over time, will likely contribute the growth of a given scientist's H-index. Holding the $\mathrm{H}$-index constant, the higher the $\mathrm{SeTH}$, the greater potential for $\mathrm{H}$-index growth in the near-term. Differences between an individual's $\mathrm{H}$-index rank and the same person's SeTH rank (referred to below as "H-Rank-minusSeTH-Rank") might be analyzed in a future study to give some indication of a given scientist's likely future movement within the $\mathrm{H}$-index ranking. Assuming that $\mathrm{SeTH}$ is a good estimation for future $\mathrm{H}$-index growth, the size of a given investigator's H-Rank-minus-SeTHRank disparity as well as it's "polarity" (i.e., whether the number is negative or positive) should predict future movement up or down in the $\mathrm{H}$-index ranking. An investigator with a highly positive H-Rank-minusSeTH-Rank disparity will be expected to move up in the H-index ranking over time since that investigator's "minor-league" papers are more highly-cited as a group than those of the people immediately above them in the current $\mathrm{H}$-index ranking. On the other hand, individuals with a significantly negative H-Rank-minus-SeTHRank should expect downward movement in the AD $\mathrm{H}$-index ranking as their minor-league papers are not as competitive as those of the people directly beneath them in today's H-index ranking. Finally, those with an H-Rank-minus-SeTH-Rank of zero can expect to stay at their current spot in the rankings for the foreseeable future assuming that there are not upwardly-mobile scientists directly below them who are poised to displace them (see Table 5 for H-minus-SeTH scores for the top-100 H-indices).

\section{Using SeTH and sfH to discriminate between similar (or identical) $\mathrm{H}$-indices}

Using the Dickson-Masters-Hardy H-index tie (all have an AD H-index of 65) as a test case, SeTH and sfH can be used to understand differences among investigators with the same $\mathrm{H}$-index.

If one wanted to predict which of the three investigators was most likely to break the $\mathrm{H}$-index tie through upward mobility in the next ranking, one approach would be to calculate the SeTH of each investigator (32,
43, and 37 respectively). Based on these numbers, it would appear that Masters is poised for much stronger $\mathrm{H}$-index growth than the other two and will likely move ahead of them in future $\mathrm{H}$-index rankings.

If, on the other hand, there was interest in determining school-of-thought leadership in a given line of investigation, then the sfH can be applied as a distinguishing factor in this area. Using the same investigators, we can apply the sfH to Dickson and Hardy, both of whom have "tau Proteins" as their main line of investigation. By comparing their sfH scores (24 and 37 respectively), it is clear that while both investigators have achieved similar overall scientific impact (i.e., the same $\mathrm{H}$-index) working in similar research areas, Hardy's impact is much more centered around a group of scientists who closely follow his work and who cite it repeatedly in their own work over an extended period time. The scientific impact of Dickson, on the other hand, while equally as great (as measured only by the $\mathrm{H}$-index), is spread out among a wider number of scientists, many of whom only cite his work sporadically in theirs. In others words, based on the comparison of their Scientific-Following $\mathrm{H}$-indices, one could easily argue for Hardy (over Dickson) to be awarded a hypothetical school-of-thought-leadership prize within AD research.

\section{AD awards}

When assessing the impact of $\mathrm{AD}$ researchers on their field, another parameter to be considered is the bestowing of research awards upon AD scientists. The two principal awards in this field are the MetLife Alzheimer Award for Medical Research and the Potamkin Prize for Research in Pick's, Alzheimer's, and Related Diseases. Table 5 indicates which scientists have won these awards. It also indicates membership in the Institute of Medicine of the US National Academy of Sciences.

Due to its subjective or "soft" nature, it is unclear how, exactly, to utilize the award-related data to further refine a bibliometric analysis of the impact of the top AD scientists. The information is presented here for completeness rather than as an integral part of the analysis. One observation, however, is that while a fair percentage of the top AD researchers have been honored with one or more award/membership, there are multiple cases of top-ranked investigators who have never been honored. Similarly, there are various instances of award-winners who do not fair exceptionally well under bibliometric analysis. One explanation for this 


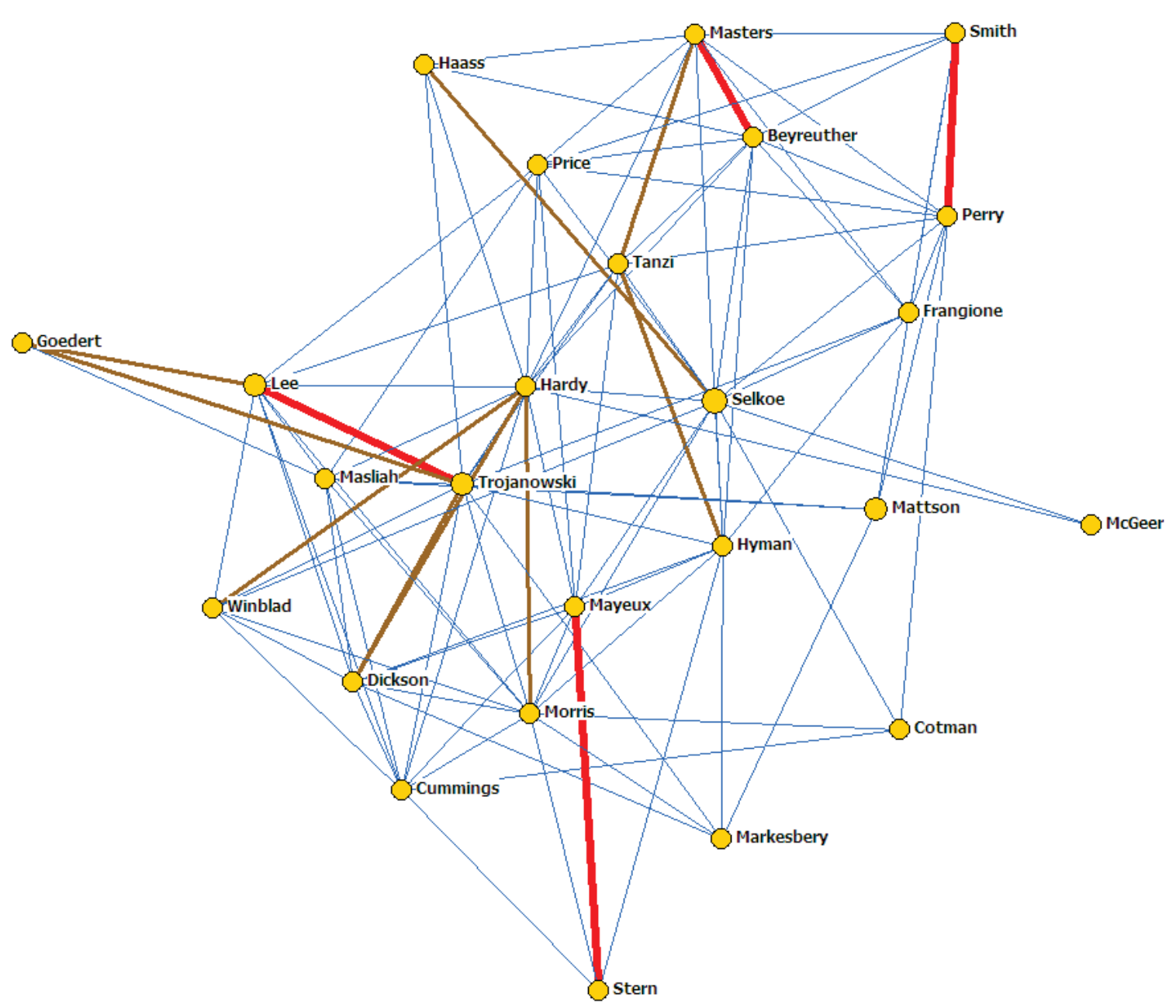

Fig. 2. Co-authorship network for top $25 \mathrm{AD}$ investigators (based on H-index). Blue $=1-10$ papers written together ( 1 pixel wide); Brown $=11-40$ papers written together ( 3 pixels wide); Red $=41-370$ papers written together ( 5 pixels wide). (Colours are visible in the electronic version of the article at www.iospress.nl.)

might be the existence of social factors in addition to scientometric criteria being used as inputs to the awardgranting process. Another possible explanation of the variation between the award-granting process and a bibliometric analysis of the top scientists is the fact that a bibliometric analysis measures an individual's total contributions over an extended period of time, while a prize might be awarded to a given scientist for a single discovery that has proven to be important or promises to be important to the field.

\section{Collaboration among Top 25}

The final phase of the scientometric analysis of the top AD scientist was a co-author analysis of the top 25
AD investigators based on data extracted from Thomson WoS and biomedexperts.com. Figure 2 is a simple network view showing strength of collaboration among the top twenty-five AD investigators. The total area of each investigator's circle corresponds to that scientist's H-index.

One point of interest is that the four, two-person coauthor teams connected by the red lines represent partnerships that are highly productive: the average number of papers per team (papers where both investigators are co-authors) is 255.5 for a total of 1022 teamwritten papers for the eight scientists. These co-author "duos" (i.e., Masters \& Beyreuther, Smith \& Perry, Lee \& Trojanowski, and Mayeux \& Stern) also generate significant impact with each team's shared papers averaging 14,610 total citations for at total of 58,441 
team-generated citations for the group of eight scientists. The phenomenon of high-impact co-authorship teams observed in the AD research community corresponds to the findings of scientometric studies of other fields [15].

\section{CONCLUSIONS}

The spirit of Lotka's law [16], which suggests that in any given scientific field the majority of the work is done by a small percentage of the total investigators, seems to hold true in AD not only in terms of the quantity of research but also in terms of the quality of the science. There are two interesting directions for future research that naturally follow the work presented in this paper. The first involves conducting a similar, but more granular, analysis of scientific leadership within the $\mathrm{AD}$ research community in which the identification of excellence within the principal AD lines of investigation is emphasized. The second future direction is that of exploring further the Second-Tier H-index and the Scientific-Following $\mathrm{H}$-index to better understand the temporal dynamics of AD research activity. This would allow one to make predications as to which established investigators are peaking in terms of their productivity and impact and which Young Turks are poised to revolutionize the scientific approaches of the mainstream AD research community.

\section{ACKNOWLEDGMENTS}

The author would like to acknowledge the contributions of the Collexis technology leadership team, namely Georg Lambert, Mario Diwersy, and Martin Schmidt. Without their author-disambiguation algorithms and bibliometric analysis platforms, this paper would not have been possible. Gagik Baghdasaryan also provided essential support in the use of the MDLogix VisuaLyzer platform used for the collaboration network visualization in Fig. 2.

\section{REFERENCES}

[1] Perry G, Knight R, Smith MA (2006) Maladie d'alzheimer: Les quarante derniers années. Alzheimer Actualités Novembre-Décembre(189), 8-11.

[2] Neuroscience Journal Citation Report [Internet]: Thomson ISI; c2003. Available from: http://admin-apps.isiknowledge. com/JCR/JCR?RQ=LIST_SUMMARY_JOURNAL\&cursor= 1.

[3] in-cites - The 100 Most-Cited Scientists in Neuroscience [Internet]: Thomson ISI; c2007 [cited 2008 9/16/2008]. Available from: http://www.in-cites.com/nobel/2007-neu-top100.html.

[4] Alzheimer's Disease Top-20 Author Report [Internet]: Thomson ISI; c2002 [cited 2008 9/16/2008]. Available from: http://www.esi-topics.com/alzheimer/authors/b1a.html.

[5] Hirsch JE (2005) An index to quantify an individual's scientific research output. Proc Natl Acad Sci U S A 102, 16569-16572.

[6] Hirsch JE (2007), Does the H index have predictive power? Proc Natl Acad Sci U S A 104, 19193-19198.

[7] Collexis-Thomson Web of ScienceDashboard - Alzheimer's Disease [Internet]: Collexis, Inc.; c2008 [cited 2008 9/ 16/2008]. Available from: http://thomson.collexis.com/ alzheimer/index.asp.

[8] Collexis-Thomson Web of Science Dashboard Description [Internet]: Collexis, Inc.; c2008 [cited 2008 9/16/2008]. Available from: http://collexis.com/products/ThomsonDashboard. htm.

[9] BiomedExperts Database [Internet]: Collexis, Inc.; c2008 [cited 2008 9/16/2008]. Available from: http://www. biomedexperts.com/.

[10] Burrell QL (2007) On the h-index, the size of the hirsch core and jin's A-index. J Informetrics 1, 170-177.

[11] MetLife Foundation Alzheimer Disease Awards [Internet]: MetLife; c2005 [cited 2008 9/16/ 2008]. Available from: http: //www.metlife.com/WPSAssets/23802966201153768910V1F 2006AwardsBrochure.pdf.

[12] Potamkin Prize for Research in Pick's, Alzheimer's, and Related Diseases - American Academy of Neurology [Internet]: American Academy of Neurology; c2008 [cited 2008 9/16/2008]. Available from: http://www.aan.com/science/ awards/?fuseaction=home.info\&id=13.

[13] MDLogix VisuaLyzer Description [Internet]: MDLogix; c2008 [cited 2008 10/7/2008]. Available from: http://mdlogix. com/solutions/additional.html\#row1.

[14] Collexis Mediator Description [Internet]: Collexis, Inc.; c2008 [cited 2008 9/17/2008]. Available from: http://collexis. com/products/mediator.htm.

[15] Borner K, Dall'Asta L, Ke W, Vespignani A (2005) Studying the emerging global brain: Analyzing and visualizing the impact of co-authorship teams. Complexity 10, 57-67.

[16] Lotka AJ (1926) The frequency distribution of scientific productivity. $J$ Washington Acad Sci 16, 317-324. 